\title{
Anabases
}

ANABASES Traditions et réceptions de l'Antiquité

$25 \mid 2017$

Varia

\section{JANKOWIAK et F. MONTINARO, Studies in Theophanes}

\section{Olivier Gengler}

\section{OpenEdition}

Journals

Édition électronique

URL : http://journals.openedition.org/anabases/6142

DOI : 10.4000/anabases.6142

ISSN : 2256-9421

\section{Éditeur}

E.R.A.S.M.E.

\section{Édition imprimée}

Date de publication : 1 avril 2017

Pagination : 290-292

ISSN : 1774-4296

\section{Référence électronique}

Olivier Gengler, « M. Jankowiak et F. montinaro, Studies in Theophanes », Anabases [En ligne], 25 | 2017, mis en ligne le 01 avril 2017, consulté le 20 janvier 2021. URL : http://journals.openedition.org/ anabases/6142 ; DOI : https://doi.org/10.4000/anabases.6142

Ce document a été généré automatiquement le 20 janvier 2021.

(c) Anabases 


\title{
M. JANKOWIAK et F. MONTINARO, Studies in Theophanes
}

\author{
Olivier Gengler
}

\section{RÉFÉRENCE}

M. JANKOWIAK et F. MONTINARO, Studies in Theophanes

Paris, Association des Amis du Centre d'Histoire et Civilisation de Byzance, 2015,

Travaux et mémoires 19, XII + 516 p.,

90 euros / ISBN 978-2-916716-58-9

1 C'est un livre important que donnent ici M. Jankowiak et F. Montinaro, la première synthèse sur la Chronique attribuée à Théophane le Confesseur († 817 ou 818), vingt ans après la traduction anglaise commentée de C.Mango et R. Scott (The Chronicle of Theophanes Confessor: Byzantine and Near Eastern history AD 284-813, Oxford, 1997), qui avait renouvelé les bases du débat scientifique autour de cette œuvre majeure, source essentielle pour l'histoire des premiers siècles du Moyen Âge byzantin.

2 La cohérence de ce volume collectif, issu d'un colloque organisé à Paris par les éditeurs à l'automne 2012, est tout à fait remarquable. Les vingt-quatre contributions qui le composent sont regroupées en ensembles thématiques traitant de l'auteur $(1:$ « The authorship of the Chronicle »), de la transmission - au sens large - de l'œuvre (2 : "Transmission, transcription, translation ») et de son contenu historique (3: " Theophanes and early byzantine history », $5:$ "Theophanes and recent history »). Un ensemble de chapitres s'intéresse en outre à la source orientale de Théophane pour les événements des $\mathrm{VII}^{\mathrm{e}}$ et $\mathrm{VIII}^{\mathrm{e}} \mathrm{s}$., qu'il partage entre autres avec la chronique de Michel le Syrien, et dans laquelle Lawrence I. Conrad (« Theophanes and the Arabic historical tradition: some indications of intercultural transmission ", Byz. Forsch., 15, 1990, p.1-44) a proposé de reconnaître l'ouvrage de Théophile d'Édesse (†785) (4: "Theophilus of Edessa »). Pour être cohérent, le volume n'en est pas pour autant univoque et des points de vue opposés sont défendus sur plusieurs questions cruciales, 
comme par exemple l'identification de l'auteur et le rôle plus ou moins grand du travail préparatoire de Georges le Syncelle dont Théophane poursuit la Chronique pour les années 284-813. Ce débat transparaît dans l'ensemble du volume, mais est plus particulièrement traité dans la première section. Après $\mathrm{C}$. Mango, Warren Treadgold ne reconnaît à Théophane qu'un rôle secondaire dans la composition de la Chronique, qui devrait presque tout à Georges le Syncelle. Dans sa contribution, Treadgold entreprend de reconstituer la vie et la carrière de ce dernier et montre qu'il avait les qualités requises pour préparer le matériau à partir duquel, après sa mort, Théophane aurait compilé sa Chronique ("The life and wider significance of George Syncellus », p. 9-30, reprenant en partie la matière de $\mathrm{W}$. Treadgold, The middle Byzantine historians, New York 2013, p. 38-63). À l'opposé, Constantin Zuckerman attribue un rôle majeur à Théophane, qu'il n'identifie cependant pas à l'abbé de Megas Agros, comme le veut le titre donné dans les manuscrits. Quoi qu'il en soit, il demeure pertinent de chercher à distinguer les éléments propres à la rédaction finale de l'œuvre de ceux relevant du travail de Georges le Syncelle, à quoi s'emploient Marek Jankowiak et Andrzej Kompa (" Framing universal history: Syncellus' canon and Theophanes' rubrics », p. 53-72 et "In search of Syncellus' and Theophanes' own words: the authorship of the Chronographia revisited ", p. 73-92 respectivement). L'article de Lee Mordechai, intégré à la dernière section de l'ouvrage en raison de sa thématique, aborde également de front cette question et met en exergue, par une analyse statistique, le rôle spécifique de Théophane - quelle que soit son identité (" The last century of Theophanes' Chronicle : a statistical approach ", p.445-466). Formant transition avec la section suivante, la contribution de Jesse Torgerson étudie la tradition manuscrite conjointe des chroniques de Georges le Syncelle et de Théophane qui tend à démontrer que ces œuvres ont été d'emblée lues comme un tout, ce qui renforce l'hypothèse d'une forte dépendance entre les deux auteurs (" From the many, one? The shared manuscripts of the Chronicle of Theophanes and the Chronography of Synkellos ", p. 93-117). Dans la seconde section, Filippo Ronconi, qui étudie aussi l'Oxoniensis Christ Church Wake 5 et le Vaticanus gr. 155, revient sur la date du ms. Parisinus gr. 1710, qu'il tend à placer dans le troisième quart du $\mathrm{IX}^{\mathrm{e}} \mathrm{s}$. et dont il suppose qu'il contenait originellement aussi le texte de Georges le Syncelle (" La première circulation de la "Chronique de Théophane ": notes paléographiques et codicologiques ", p.121-147). Les contributions suivantes s'intéressent particulièrement à la diffusion de la Chronique et à sa réception (Bronwen Neil, "Theophanes Confessor on the Arab conquest : the Latin version by Anastasius Bibliothecarius ", p. 149-157 ; Juan Signes Codoñer, "Theophanes at the time of Leo VI », p. 159-176, qui suppose que l'Ox. Christ Church Wake 5 et le Vat. gr. 155 représentent une seconde édition de la Chronique datant du tout début du Xe s. ; Federico Montinaro, "The Chronicle of Theophanes in the indirect tradition ", p.177-205; Anna-Marija Totomanova, "The Chronicle of Theophanes the Confessor in the Slavic tradition ", p. 207-235). Les sources de Théophane sont au cœur de la majorité des contributions des sections ultérieures. La comparaison de la Chronique de Théophane avec certains textes conservés qui ont pu lui servir directement ou indirectement permettent d'apprécier la manière dont l'auteur a travaillé son texte. Synthétisant ses précédentes contributions sur le sujet, Roger Scott revient ainsi sur la manière dont Théophane utilise Malalas : bien qu'il ait disposé d'une version manifestement plus complète que celle transmise par la tradition directe, Théophane, par la sélection qu'il opère et les modifications qu'il apporte au texte de Malalas, propose bien sa propre vision du passé ("The first half of Theophanes' Chronicle », p. 239-260). Anna Kotłowska et Łukasz 
Różycki montrent qu'il en est de même pour la manière dont Théophane exploite les less Histoires de Théophylacte Simocatta (" The battle of Solachon of 586 in light of the works of Theophylact Simocatta and Theophanes ", p. 315-326). Bernard Pouderon explore pour sa part les relations entre Théophane et l'Histoire ecclésiastique de Théodore le Lecteur, soulignant le rôle spécifique de l'épitomé de celle-ci et d'une source possible de Théodore et de son épitomateur, que Théophane aurait pu utiliser également: Jean Diacrinoménos ("Théophane, témoin de l'Épitomé d'histoire ecclésiastique de Théodore le lecteur ou de Jean Diacrinoménos ? ", p. 279-314). Dans une perspective classique de Quellenforschung, plusieurs autres contributions explorent les possibilités d'identifier les sources perdues sur lesquelles se serait appuyé Théophane (Geoffrey Greatrex, «Théophane et ses sources sur la guerre d'Anastase I ${ }^{\text {er }}$ contre les Perses », p. 269-278 ; Stephanie Forrest, « Theophanes' Byzantine source for the late seventh and early eighth centuries, c. AD 668-716 ", p. 417-444 ; Dmitry Afinogenov, "Style, structure, and authorship of the hypothetical source of Theophanes for the reigns of Leo III and Constantine V », p.467-472 ; et la section consacrée à Théophile d'Édesse et les sources orientales de Théophane : Robert G. Hoyland, "Agapius, Theophilus and Muslim sources ", p.355-364 ; Muriel Debié, "Theophanes' "Oriental source" : what can we learn from Syriac historiography? ", p. 365-382 ; Maria Conterno, "Theophilos, "the more likely candidate"? Towards a reappraisal of the question of Theophanes' "Oriental source(s)" ", p. 383-400 ; Andy Hilkens, "Before the Eastern source: Theophanes and the late Syriac Orthodox chronicles, $4^{\text {th }}-6^{\text {th }}$ centuries ", p. 401-413). James Howard-Johnston montre enfin que, pour les événements les plus récents, l'information de Théophane dérive de communiqués officiels, ce qui explique la précision de son récit, mais n'en augmente pas pour autant l'objectivité (" Theophanes on the recent past : the crisis of 782 and its antecedents ", p. 473-490). Les autres contributions traitent de la manière dont Théophane aborde dans son œuvre la vénération des reliques (Irina Tamarkina, "Veneration of relics in the Chronicle of Theophanes ", p. 261-267) et les réalités que, d'un point de vue moderne, on peut rattacher à l'économie (Salvatore Cosentino, " La perception du domaine économique dans la Chronographie de Théophane ", p. 327-352). Des index des sources, des manuscrits et des anthroponymes et toponymes permettent de s'orienter dans cet ouvrage dense et stimulant qui jette les bases de toute nouvelle discussion sur Théophane et sa Chronique.

\section{AUTEURS}

\section{OLIVIER GENGLER}

Académie des Sciences de Heidelberg / Université de Tübingen

ogengler@yahoo.fr 